\title{
Phenomenological study of the atypical heavy flavor production observed at the Fermilab Tevatron
}

\author{
G. Apollinari, ${ }^{2}$ M. Barone, ${ }^{1}$ I. Fiori, ${ }^{3}$ P. Giromini, ${ }^{1}$ \\ F. Happacher, ${ }^{1}$ S. Miscetti, ${ }^{1}$ A. Parri, ${ }^{1}$ and F. Ptohos ${ }^{4}$ \\ ${ }^{1}$ Laboratori Nazionali di Frascati, Istituto Nazionale di Fisica Nucleare, Frascati, Italy \\ ${ }^{2}$ Fermi National Accelerator Laboratory, Batavia, Illinois 60510, USA \\ ${ }^{3}$ Istituto Nazionale di Fisica Nucleare, I-56100 Pisa, Italy \\ ${ }^{4}$ University of Cyprus, 1678 Nicosia, Cyprus
}

\begin{abstract}
We address known discrepancies between the heavy flavor properties of jets produced at the Tevatron collider and the prediction of conventional-QCD simulations. In this study, we entertain the possibility that these effects are real and due to new physics. We show that all anomalies can be simultaneously fitted by postulating the additional pair production of light bottom squarks with a $100 \%$ semileptonic branching fraction.
\end{abstract}

PACS numbers: $13.85 . \mathrm{Qk}, 13.20 . \mathrm{He}, 12.60 . \mathrm{Jv}$ 


\section{INTRODUCTION}

In 2001 the CDF experiment reported [1] the observation of an excess of events - containing a lepton with large transverse momentum, large transverse missing energy, and two or three jets one of which (called superjet) contains a displaced secondary vertex and a soft lepton due to the decay of presumed $b$ hadrons - over the standard model (SM) expectation (17 such events are observed and $4.9 \pm 0.6$ are expected $)^{1}$.

The apparent excess of superjets observed by CDF has been modeled in Ref. [3] by postulating the unconventional production of a low-mass, strongly interacting object that decays semileptonically with a branching fraction of the order of 1 and a lifetime of the order of the picosecond. Since at the time there were no limits to the existence of a charge $-1 / 3$ scalar quark lighter than $10 \mathrm{GeV} / \mathrm{c}^{2}[4-6]$, the supersymmetric partner of the bottom quark was a potential candidate. The hypothesis of a very light scalar quark has been investigated in many theoretical papers and has prompted careful re-examinations of precision electroweak data and $e^{+} e^{-} \rightarrow$ hadrons data [7-21]. It is a fair summary of all this work that no data analysis shows convincing evidence for a very light scalar quark. The conjecture itself is not favored, but also not completely excluded, in the framework of the minimal supersymmetric standard model (MSSM). The CLEO collaboration [22] has searched for pair production of light squarks that decay to a lepton, a charmed quark and a scalar neutrino with a $100 \%$ branching fraction by selecting events that contain two oppositely charged leptons plus a $D$ meson below the $B \bar{B}$ threshold. Reference [22] reports no evidence for the production of such squarks with mass between 3.5 and $4.5 \mathrm{GeV} / \mathrm{c}^{2}$. However, an exclusion limit depends on the modeling of the scalar quark hadronization and decay matrix element, and squarks lighter than $3.8 \mathrm{GeV} / \mathrm{c}^{2}$ could have escaped detection [22].

Because of the unresolved experimental situation, the CDF collaboration has performed a detailed comparison [23] between measured and predicted heavy-flavor properties of jets produced at the Tevatron, intended to search for evidence either supporting or disfavoring this conjecture. Reference [23] studies rates of observed and predicted leptons arising from

${ }^{1}$ The D $\varnothing$ collaboration has also searched [2] for the presence of such an anomaly, and reports a deficit with respect to the SM prediction ( 2 events observed and $3 \pm 0.5$ predicted). Unfortunately, because of stricter selection requirements and a soft lepton tagging efficiency significantly smaller than that of CDF, the resulting $95 \%$ C.L. limit to the cross section for producing this type of events is at least a factor of two larger than the cross section corresponding to the excess observed by CDF [3]. 
$b$-quark decays in jets that recoil against a generic jet or recoil against a jet that also contains a lepton. That study finds that in the second case the rate of jets containing a lepton from presumed $b$ decays is $50 \%$ higher than in the first case. The magnitude of the effect is consistent with what is expected in the presence of pair production of light squarks with a $100 \%$ semileptonic branching ratio. The magnitude of the discrepancy is consistent with the result of other measurements performed at the Tevatron. The ratio of the $\mu+\bar{b}$-jet cross section to the next-to-leading-order (NLO) prediction ${ }^{2}$ is measured to be $1.5 \pm 10 \%$ [24], the ratio of the cross section for producing dimuons from presumed $b$ - and $\bar{b}$-hadron semileptonic decays to the NLO prediction is $2.6 \pm 20 \%[25,26]$, whereas this ratio is $1.1 \pm 20 \%$ when identifying $b$ quarks by locating secondary vertices produced by their decays $[23,27,28]$. If one assumes that the above mentioned measurements and their quoted uncertainties are correct, the disagreement between data and theory appears to be a function of the number of semileptonic decays used to identify $b$ quarks ${ }^{3}$. Reference [29] has extended the comparison of Ref. [23] to jets containing a lepton pair. In that study, the distributions of the dilepton invariant mass and opening angle are found to be strikingly different from those predicted by a conventional-QCD simulation, in which most lepton pairs arise from sequential semileptonic decays of single $b$ hadrons. In addition, a study of the invariant mass distribution of muon pairs collected by the CDF experiment [30] has attempted to rule out $1^{--}$bound states of light scalar quarks that are not excluded by the SPEAR searches for narrow resonances [5,31]. Reference [30] improves the SPEAR limit by almost an order of magnitude, and sets an average upper credible limit of approximately $8 \mathrm{eV}$ to the leptonic width of new narrow resonances below the $\Upsilon$ mesons. An exception is the mass of $7.2 \mathrm{GeV} / \mathrm{c}^{2}$ at which an excess of $250 \pm 61$ events over a smooth background is observed. The statistical significance of the signal $(3.5 \sigma)$ is not enough to establish the existence of a new particle. However, the size of this signal is consistent with the production of a $1^{--}$bound state of spin 0 and charge $-1 / 3$ quarks.

All anomalies listed above certainly require further and independent confirmation. How-

${ }^{2}$ As discussed in Ref. [23], the NLO prediction for producing two central bottom quarks above the same transverse momentum threshold has an uncertainty no larger than $15 \%$; in contrast, the NLO prediction of the single $b$ cross section has $\mathrm{a} \simeq 50 \%$ uncertainty.

3 This effect could also be a fortuitous result produced by the fact that the distribution of the ratios of the $b \bar{b}$ cross section measurements at the Tevatron, with and without leptons, to NLO prediction has approximately a $30 \%$ RMS deviation, considerably larger than the quoted experimental uncertainties [28]. 
ever, we entertain the possibility that all these effects are real, and verify if a new physics process can simultaneously model the discrepancies between data and conventional-QCD predictions reported in Refs. [23, 29]. Since we use the same data sets, simulated samples, and analysis tools we briefly recall those studies. Reference [23] compares a QCD simulation based upon the HERWIG [32] and QQ [33] Monte Carlo generator programs to a data sample, referred to as inclusive lepton sample, that consists of events with two or more jets, one of which is required to contain a lepton with $p_{T} \geq 8 \mathrm{GeV} / \mathrm{c}$ (trigger lepton); this jet is referred to as lepton-jet, whereas jets recoiling against it are called away-jets. That study uses measured rates of lepton- and away-jets containing displaced secondary vertices (SECVTX tags) or tracks with large impact parameter [34] (JPB tags) in order to determine the bottom and charmed content of the data. In order to remove the uncertainty of the predicted heavy flavor production cross sections, the simulation is tuned to match the heavy-flavor content of the data. In Ref. [23] rates of SECVTX and JPB tags, as well as the event kinematics, are well modeled after tuning the parton-level cross sections predicted by the HERWIG generator within the experimental and theoretical uncertainties. However, the data have a $50 \%$ excess of events in which one additional jet (away-jet) contains a soft lepton with $p_{T} \geq 2 \mathrm{GeV} / \mathrm{c}$ (SLT tag). Reference [29] has extended the comparison between data and tuned simulation to lepton-jets that, in addition to the trigger lepton, contain an additional soft lepton with opposite sign charge (OS). After removing the background arising from fake leptons by subtracting rates of lepton pairs with same sign charge (SS), the yields of OS-SS lepton pairs are compared to the tuned simulation. For invariant masses smaller than $2 \mathrm{GeV} / \mathrm{c}^{2}$, the data are found to be largely underestimated by the simulation.

In the present study we try to model the same data used in Refs. [23, 29] by implementing the conventiona-QCD simulation, referred to as SM simulation in the following, with the hypothetical pair production of sbottom quarks $\left(b_{s}\right)$. Section II describes the implementation of this new process into the SM simulation. In Sec. III we tune the $\mathrm{SM}+b_{s}$ simulation to also reproduce measured yields of jets with SLT and dilepton tags. Section IV investigates the sensitivity of the data to other models. Section V presents a crude estimate of the lifetime of the hypothetical object causing the excess of SLT tags. Our conclusions are presented in Sec. VI. 


\section{PRODUCTION AND DECAY OF SBOTTOM QUARKS}

The production of $b_{s}$ quarks that decay to $l X$ with a $100 \%$ branching ratio would be sufficient to model the excess of away-jets with SLT tags reported in Ref. [23]. In order to model the excess of jets containing a lepton pair reported in Ref. [29], we need an extra source of leptons. This could be provided by the sequential semileptonic decay of $c$ quarks, and we choose the decay $b_{s} \rightarrow c l \nu_{s}$, where $\nu_{s}$ is a massless non-interacting scalar.

We model the $b_{s}$ decay with the matrix element discussed in more detail in Appendix A. In this model, the decay is mediated by the higgsino coupling to the right-handed matter. This matrix element produces lepton pairs from sequential decay that have an invariant mass distribution different from that produced by the $V-A$ matrix element of conventional heavy-flavor decays (see Fig. 1). We implement the production of pairs of scalar quarks in $p \bar{p}$ collisions into the HERWIG generator with the method described in Appendix B. The method starts with the generation of $b \bar{b}$ pairs with a quark mass of $3.65 \mathrm{GeV} / \mathrm{c}^{2}$ and a lifetime of 1 ps. At the end of the $b$-hadronization process, the formed $B$ hadrons are turned into fictitious $\tilde{B}$ hadrons. We have generated samples of simulated events with the same luminosity of the conventional-QCD samples used in Refs. [23, 29]. The NLO calculation of the process $p \bar{p} \rightarrow b_{s} \bar{b}_{s}$, implemented into the PROSPINO Monte Carlo generator [35], predicts a cross section that is approximately $15 \%$ of the NLO cross section for producing pairs of quarks with the same mass [36]. Therefore the parton-level $b_{s}$ cross section in the HERWIG simulated samples is overestimated by a factor of seven. 

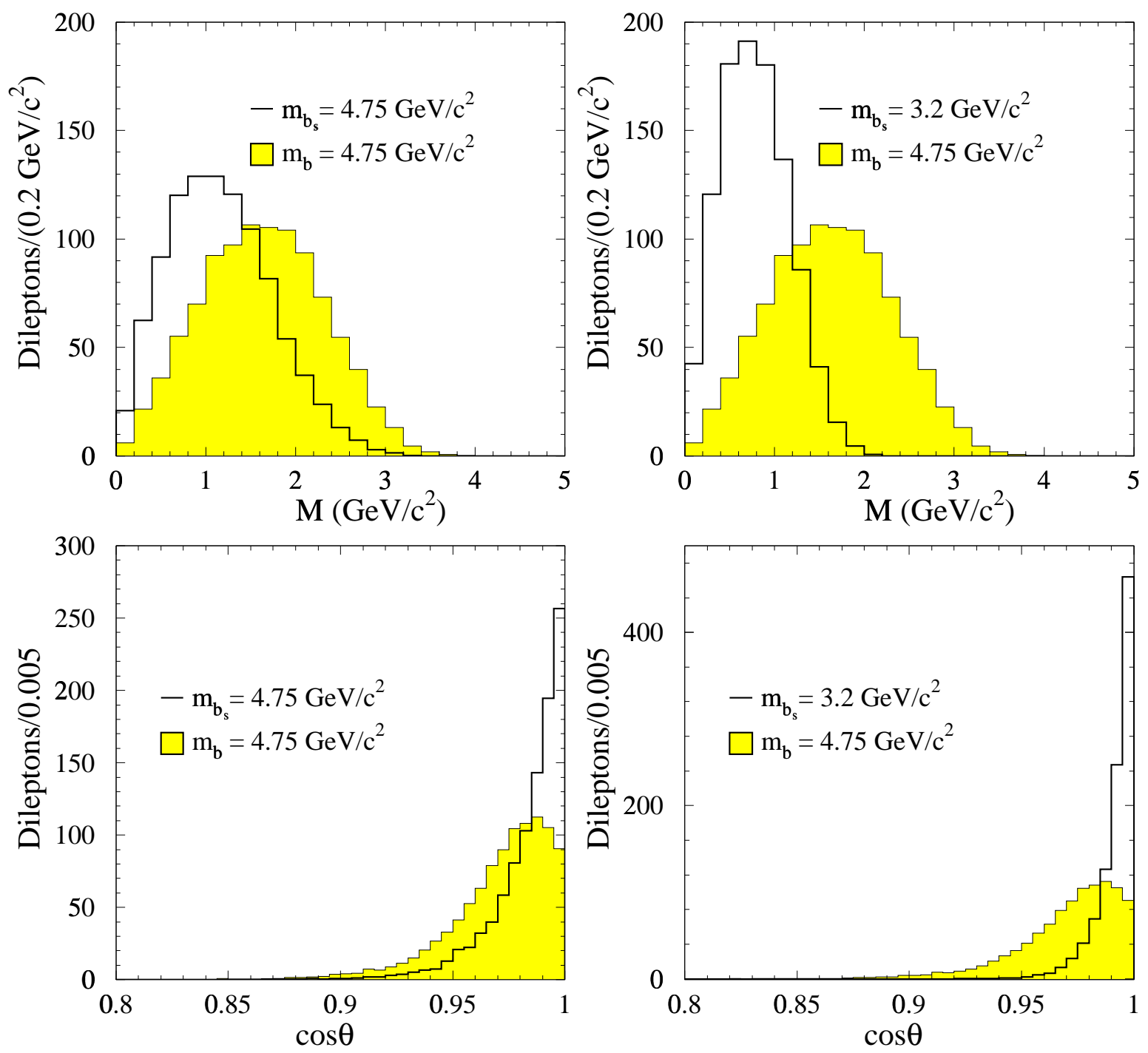

FIG. 1: Distributions of the invariant mass $M$ and the opening angle $\theta$ between leptons arising from the sequential decays $b \rightarrow l c \nu$, with $c \rightarrow l s \nu$ and $b_{s} \rightarrow l c \nu_{s}$, with $c \rightarrow l s \nu$. We set $m_{c}=1.5 \mathrm{GeV} / \mathrm{c}^{2}$. Leptons are not simulated through the detector. 


\section{FIT OF THE DATA WITH THE SM SIMULATION IMPLEMENTED WITH PAIR PRODUCTION OF SCALAR QUARKS}

In Ref. [23], after tuning the SM simulation to fit the observed rates of lepton- and away-jets with SECVTX and JPB tags due to heavy flavor, we have observed a discrepancy between measured and predicted rates of away-jets with soft lepton tags. In this study, we use a simulation that includes pair production of $b_{s}$ quarks with $100 \%$ semileptonic branching ratio; we tune this production by also fitting rates of away-jets with SLT tags. Rates of tagged jets in the data are taken from Tables II and VII of Ref. [23]. We also fit rates of (OS-SS) lepton pairs ( $i l l$ ) contained in the same jet, taken from Table III of Ref. [29]. We use the same notations and fitting procedure described in Sec. VIII of Ref. [23].

In the fit we tune the parton-level cross section of the different heavy-flavor production mechanisms [direct production (LO), flavor excitation, and gluon splitting] to reproduce the rates of tags observed in the data. Following the procedure of Ref. [23], we use two fit parameters to account for the uncertainty of the luminosity and selection cuts of the electron and muon data; these factors include the uncertainty of the $b$ direct production. Five fit parameters are used to determine the relative weight of the remaining parton-level cross sections for producing conventional heavy flavors ( $b$ and $c$ ) with respect to the $b$ direct production. These five parameters are constrained to the default values as described in Sec. VIII of Ref. [23]. Direct production and flavor excitation of $b_{S}$ quarks are weighted with two additional fit parameters. As explained in Appendix B, the value of the flavor excitation cross section returned by the fit also includes gluon splitting contributions. The efficiency for finding SECVTX and JPB tags in the simulation have been tuned using a subset of the inclusive lepton sample [37]. Since we are now entertaining the hypothesis that this data sample might be contaminated by unconventional production, in the fit we also weight the $b$ - and $c$-quark tagging efficiencies with free parameters (scale factors) to allow for the possibility that they might be wrong. We assume that the simulated efficiency for tagging $b_{s}$ hadrons is correct ${ }^{4}$.

The result returned by the best fit with the $\mathrm{SM}+b_{s}$ simulation is listed in Table I. This

\footnotetext{
${ }^{4}$ We have arbitrarily fixed the $b_{s}$ lifetime; the introduction of a scale factor for the simulated $b_{s}$ tagging efficiency is equivalent to introducing an uncertainty on the lifetime. The dependence on the lifetime is studied in Sec. V.
} 
table should be compared to Table IV of Ref. [23]. From the fit parameters, we derive a cross section for producing $b_{s}$ quarks that is $(11 \pm 2) \%$ of the production cross section of $b$ quarks with the same mass, in reasonable agreement with the theoretical prediction mentioned in the previous section. Table II compares tagging rates in the data and in the simulation tuned according to the best fit. This table should be compared to Tables VI and IX of Ref. [23] and Table III of Ref. [29].

Because of the approximations in the modeling of the $b_{s}$ production and decay listed in Appendix $\mathrm{B}$, a determination of the systematic errors of the $b_{s}$ production cross section is beyond the scope and reach of this study. As an example, the HERWIG spectator model

predicts a reasonable, but also quite arbitrary, $\tilde{B}$ mass of approximately $4 \mathrm{GeV} / \mathrm{c}^{2}$. A $\pm 0.3 \mathrm{GeV} / \mathrm{c}^{2}$ change of the $\tilde{B}$ mass results in a $\pm 40 \%$ change of the $b_{s}$ production cross section returned by the best fit to the data.

Figures 2 to 4 compare observed distributions of the invariant mass and the opening angle of dileptons in the same jet to the $\mathrm{SM}+b_{s}$ simulation. The simulation is normalized with the fit parameters listed in Table I. These data appeared to be anomalous when compared to the SM simulation in Ref. [29]. For completeness, Figure 5 compares the transverse momentum distribution of dileptons in the same jet. In conclusion, the hypothesis of a light parton with a $100 \%$ semileptonic branching ratio and a production cross section typical of scalar quarks fits the yet unresolved discrepancies between the heavy flavor production observed at the Tevatron and the prediction of conventional-QCD simulations reported in Refs. [23-26, 29].

\section{SENSITIVITY OF THE DATA TO OTHER MODELS}

In the previous section we have used a $b_{s}$ mass of $3.65 \mathrm{GeV} / \mathrm{c}^{2}$. This value was chosen because, in the invariant mass spectrum of muon pairs collected by the CDF experiment, we have seen an excess of events at a mass of $7.22 \mathrm{GeV} / \mathrm{c}^{2}$ consistent with the production of a bound state of scalar quarks. Since this excess could be a statistical fluctuation, we have also generated several simulations with $b_{s}$ masses ranging from 2.5 to $5.0 \mathrm{GeV} / \mathrm{c}^{2}$. We find that the data are reasonably well modeled when using any $b_{s}$ mass between 3 and $4 \mathrm{GeV} / \mathrm{c}^{2}$.

As correctly pointed out in Ref. [9], the measured semileptonic branching fraction of $b$ 
TABLE I: Result of the fit with the SM simulation implemented with the pair production of scalar quarks $b_{s}$. The scalar quark mass is set to $3.65 \mathrm{GeV} / \mathrm{c}^{2}$ and its lifetime to $1.0 \mathrm{ps}$. The fit yields a $\chi^{2}$ of 10 for 20 degrees of freedom.

\begin{tabular}{lr}
\hline \hline SECVTX scale factor $(b$ quarks) & $0.98 \pm 0.03$ \\
SECVTX scale factor $(c$ quarks) & $1.02 \pm 0.22$ \\
JPB scale factor $(b$ and $c)$ & $1.00 \pm 0.02$ \\
$e$ norm. & $0.96 \pm 0.06$ \\
$\mu$ norm. & $0.96 \pm 0.06$ \\
$c$ dir. prod. & $1.01 \pm 0.14$ \\
$b$ flav. exc. & $0.79 \pm 0.16$ \\
$c$ flav. exc. & $0.87 \pm 0.26$ \\
$g \rightarrow b \bar{b}$ & $1.32 \pm 0.18$ \\
$g \rightarrow c \bar{c}$ & $1.36 \pm 0.34$ \\
$b_{s}$ dir. prod. & $0.07 \pm 0.03$ \\
$b_{s}$ flav. exc. + GSP & $0.20 \pm 0.04$ \\
\hline \hline
\end{tabular}

hadrons would be higher than the standard model expectation if $m_{b_{s}}+m_{\nu_{s}} \leq m_{b}{ }^{5}$. We have produced simulations of the $b_{s}$ decay with $\nu_{s}$ masses of the order of $1 \mathrm{GeV} / \mathrm{c}^{2}$. The predicted distributions for the dilepton kinematics look very similar to the ones shown in Figs. 2 to 4 ; the fit to the observed tagging rates yields a higher $b_{s}$ production cross section (17\% of that for producing $b$ quarks with the same mass).

We have chosen a $b_{s}$ semileptonic decay mediated by the right-handed component of the Higgsino coupling. We have also explored the use of a decay matrix mediated by the chargino left-handed coupling to ordinary matter (see Appendix A) and the hypothesis of a $R$-parity violating $b_{s} \rightarrow l c$ decay. Both models produce leptons with a stiffer energy spectrum than the right-handed decay mode, but also provide a fair description of the data when using $b_{s}$ masses approximately equal or slightly smaller than $3.2 \mathrm{GeV} / \mathrm{c}^{2}$. In these cases, the $b_{s}$

\footnotetext{
${ }^{5}$ In this case the decay $b \rightarrow b_{s} \bar{\nu} \nu_{s}$ with $b_{s} \rightarrow l X$ is allowed and does not suffer CKM suppression. This decay would however produce leptons with momentum significantly softer than those from direct semileptonic $b$ decays.
} 
TABLE II: Numbers of tags due to heavy flavors in the data and in the SM+bs simulation. The simulation is normalized according to the fit result listed in Table I. The SM ( $b$ and $c$ quarks) and $b_{s}$ contributions are also listed separately. The electron (muon) sample consists of events in which the trigger lepton is an electron (muon). In the data, a fraction of the trigger leptons is faked by hadrons that mimic the lepton signature: the heavy flavor purity determined by the fit is $(44.5 \pm 1.3) \%$ for the electron sample and $(58.8 \pm 2.2) \%$ for the muon sample.

\begin{tabular}{|c|c|c|c|c|c|}
\hline \multirow[b]{2}{*}{ Tag type } & \multicolumn{5}{|c|}{ Electron sample } \\
\hline & Data & Simulation & SM & $b_{s}$-dir & $b_{s}-($ f.exc + GSP $)$ \\
\hline$H F_{l-j e t}$ & 68544 & $30478.0 \pm 878.4$ & $26040.0 \pm 1282.2$ & $575.7 \pm 184.9$ & $3862.4 \pm 882.7$ \\
\hline$H F_{a-j e t}$ & 73335 & $32559.0 \pm 943.7$ & $27942.2 \pm 1354.9$ & $593.1 \pm 190.5$ & $4023.7 \pm 919.5$ \\
\hline$H F T_{l-j e t}^{S E C}$ & $10115.3 \pm 101.7$ & $10135.9 \pm 144.7$ & $8641.1 \pm 328.2$ & $189.0 \pm 61.4$ & $1305.8 \pm 302.6$ \\
\hline$H F T_{l-j e t}^{J P B}$ & $11165.4 \pm 115.8$ & $11136.1 \pm 142.1$ & $9279.5 \pm 379.7$ & $235.0 \pm 75.9$ & $1621.7 \pm 372.1$ \\
\hline$H F T_{a-j e t}^{S E C}$ & $3719.8 \pm 93.5$ & $3678.2 \pm 102.8$ & $3384.1 \pm 124.4$ & $173.8 \pm 56.5$ & $120.3 \pm 28.8$ \\
\hline$H F T_{a-j e t}^{J P B}$ & $4021.3 \pm 140.7$ & $3972.5 \pm 104.1$ & $3572.0 \pm 130.4$ & $228.2 \pm 73.7$ & $172.2 \pm 40.4$ \\
\hline$H F D T^{S E C}$ & $1375.2 \pm 37.6$ & $1373.4 \pm 55.9$ & $1279.0 \pm 59.5$ & $53.9 \pm 18.0$ & $40.5 \pm 10.7$ \\
\hline$H F D T^{J P B}$ & $1627.8 \pm 43.7$ & $1647.2 \pm 53.6$ & $1458.9 \pm 57.8$ & $89.7 \pm 29.4$ & $98.6 \pm 23.7$ \\
\hline$H F T_{a-j e t}^{S L T}$ & $862.2 \pm 114.8$ & $840.8 \pm 98.1$ & $534.7 \pm 63.0$ & $172.0 \pm 58.0$ & $134.1 \pm 34.3$ \\
\hline$H F T_{a-j e t}^{S L T \cdot S E C}$ & $322.1 \pm 23.3$ & $309.6 \pm 26.9$ & $219.8 \pm 20.7$ & $53.1 \pm 17.7$ & $36.6 \pm 9.6$ \\
\hline$H F T_{a-j e t}^{S L T \cdot J P B}$ & $349.4 \pm 26.3$ & $353.0 \pm 29.9$ & $224.5 \pm 20.1$ & $69.2 \pm 22.9$ & $59.3 \pm 14.8$ \\
\hline Dil & $1111 \pm 54.2$ & $1149.6 \pm 130.7$ & $752.2 \pm 88.0$ & $52.2 \pm 17.8$ & $345.2 \pm 86.9$ \\
\hline$D i l^{S E C}$ & $401.0 \pm 25.0$ & $436.7 \pm 35.9$ & $312.5 \pm 29.1$ & $14.2 \pm 4.9$ & $109.9 \pm 27.1$ \\
\hline \multirow[t]{2}{*}{$D i l^{J P B}$} & $464.0 \pm 26.8$ & $480.6 \pm 38.9$ & $323.1 \pm 29.0$ & $18.1 \pm 6.1$ & $139.4 \pm 33.7$ \\
\hline & \multicolumn{5}{|c|}{ Muon sample } \\
\hline Tag type & Data & Simulation & $\mathrm{SM}$ & $b_{s}$-dir & $b_{s}-($ f.exc + GSP $)$ \\
\hline$H F_{l-j e t}$ & 14966 & $8796.6 \pm 310.8$ & $7161.1 \pm 425.7$ & $234.2 \pm 75.1$ & $1401.3 \pm 311.1$ \\
\hline$H F_{a-j e t}$ & 16460 & $9557.2 \pm 335.5$ & $7831.5 \pm 456.7$ & $246.5 \pm 79.0$ & $1479.1 \pm 328.4$ \\
\hline$H F T_{l-j e t}^{S E C}$ & $3657.3 \pm 60.8$ & $3635.2 \pm 81.2$ & $2962.3 \pm 137.5$ & $94.9 \pm 30.8$ & $578.0 \pm 130.2$ \\
\hline$H F T_{l-j e t}^{J P B}$ & $4068.6 \pm 66.2$ & $4051.1 \pm 83.6$ & $3234.5 \pm 156.8$ & $115.3 \pm 37.2$ & $701.3 \pm 156.3$ \\
\hline$H F T_{a-j e t}^{S E C}$ & $943.8 \pm 35.2$ & $957.2 \pm 38.7$ & $842.3 \pm 43.5$ & $74.5 \pm 24.2$ & $40.4 \pm 9.7$ \\
\hline$H F T_{a-j e t}^{J P B}$ & $1086.8 \pm 45.0$ & $1052.0 \pm 40.0$ & $899.2 \pm 45.2$ & $95.8 \pm 30.9$ & $56.9 \pm 13.2$ \\
\hline$D T^{S E C}$ & $452.6 \pm 21.6$ & $467.4 \pm 27.6$ & $416.6 \pm 28.5$ & $30.3 \pm 10.1$ & $20.5 \pm 5.3$ \\
\hline$D T^{J P B}$ & $546.4 \pm 25.1$ & $557.3 \pm 25.5$ & $468.8 \pm 26.9$ & $46.5 \pm 15.2$ & $42.1 \pm 10.0$ \\
\hline$H F T_{a-j e t}^{S L T}$ & $271.9 \pm 34.9$ & $235.3 \pm 31.3$ & $127.6 \pm 18.2$ & $67.8 \pm 22.9$ & $39.8 \pm 10.2$ \\
\hline$H F T_{a-j e t}^{S L T \cdot S E C}$ & $63.2 \pm 10.0$ & $82.3 \pm 10.4$ & $46.8 \pm 7.5$ & $23.8 \pm 7.9$ & $11.7 \pm 3.2$ \\
\hline$H F T_{a-j e t}^{S L T \cdot J P B}$ & $103.0 \pm 12.4$ & $101.9 \pm 11.8$ & $54.9 \pm 7.7$ & $29.9 \pm 9.9$ & $17.0 \pm 4.3$ \\
\hline Dil & $336.0 \pm 28.5$ & $371.1 \pm 45.9$ & $232.9 \pm 31.6$ & $23.1 \pm 7.9$ & $120.8 \pm 29.9$ \\
\hline$D i l^{S E C}$ & $158.2 \pm 15.9$ & $168.3 \pm 17.2$ & $116.9 \pm 15.0$ & $8.0 \pm 2.7$ & $43.4 \pm 10.6$ \\
\hline$D i l^{J P B}$ & $171.5 \pm 16.5$ & $177.7 \pm 17.1$ & $115.5 \pm 13.9$ & $9.5 \pm 3.2$ & $52.7 \pm 12.6$ \\
\hline
\end{tabular}



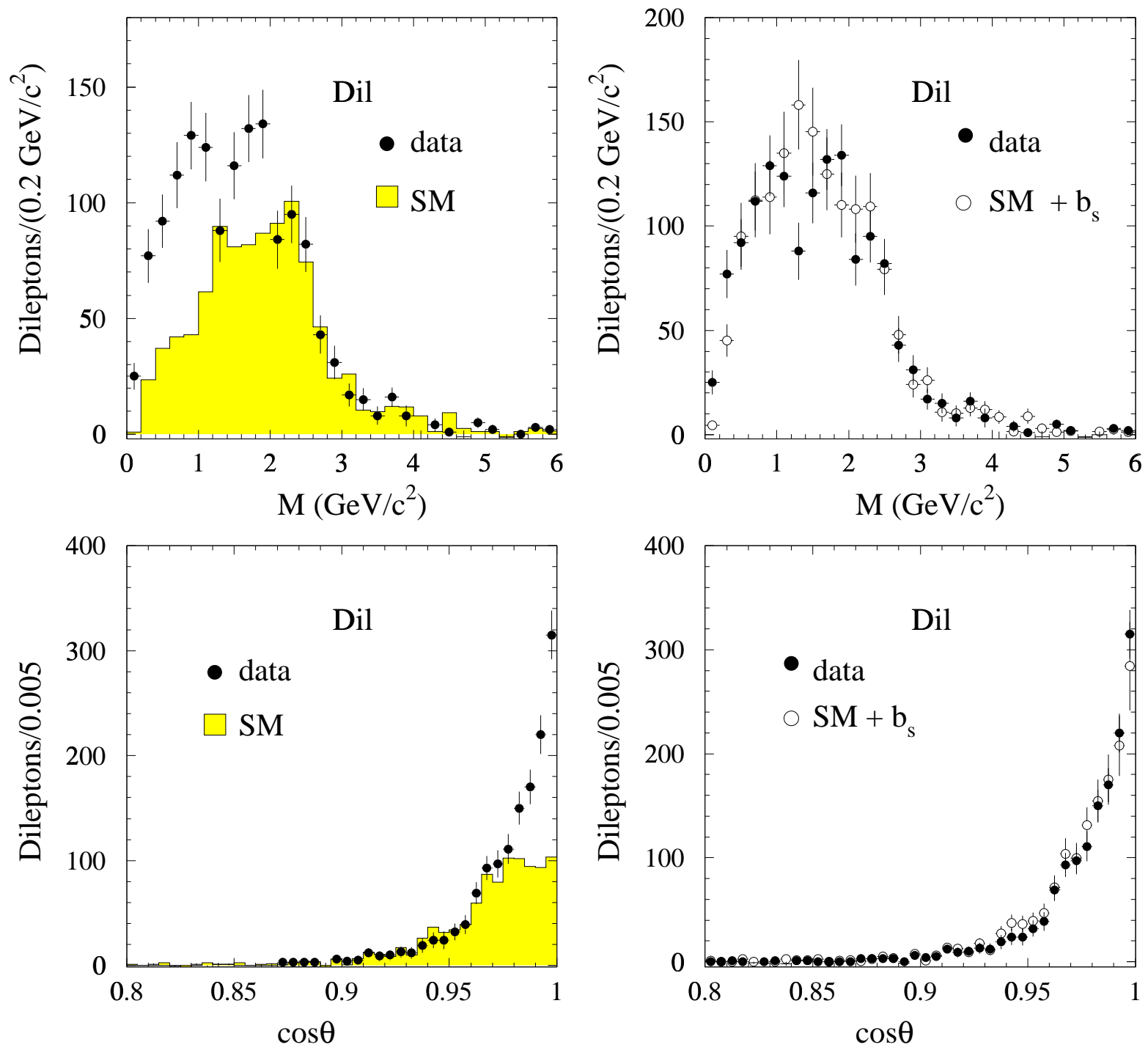

FIG. 2: Observed distributions of the invariant mass, $M$, and the opening angle, $\theta$, of dileptons in the same jet are compared to the simulation without (SM) and with $b_{s}$-quark production. The simulation is normalized with the fit parameters listed in Table I.

production cross section returned by the best fit to the observed tagging rates is $\simeq 5 \%$ of the production cross section of quarks with the same mass. We note that a sbottom quark with a mass of approximately $3.2 \mathrm{GeV} / \mathrm{c}^{2}$ is not favored by the study in Ref. [30], but is out of the mass range investigated in the CLEO analysis [22]. In the next two subsections, we 

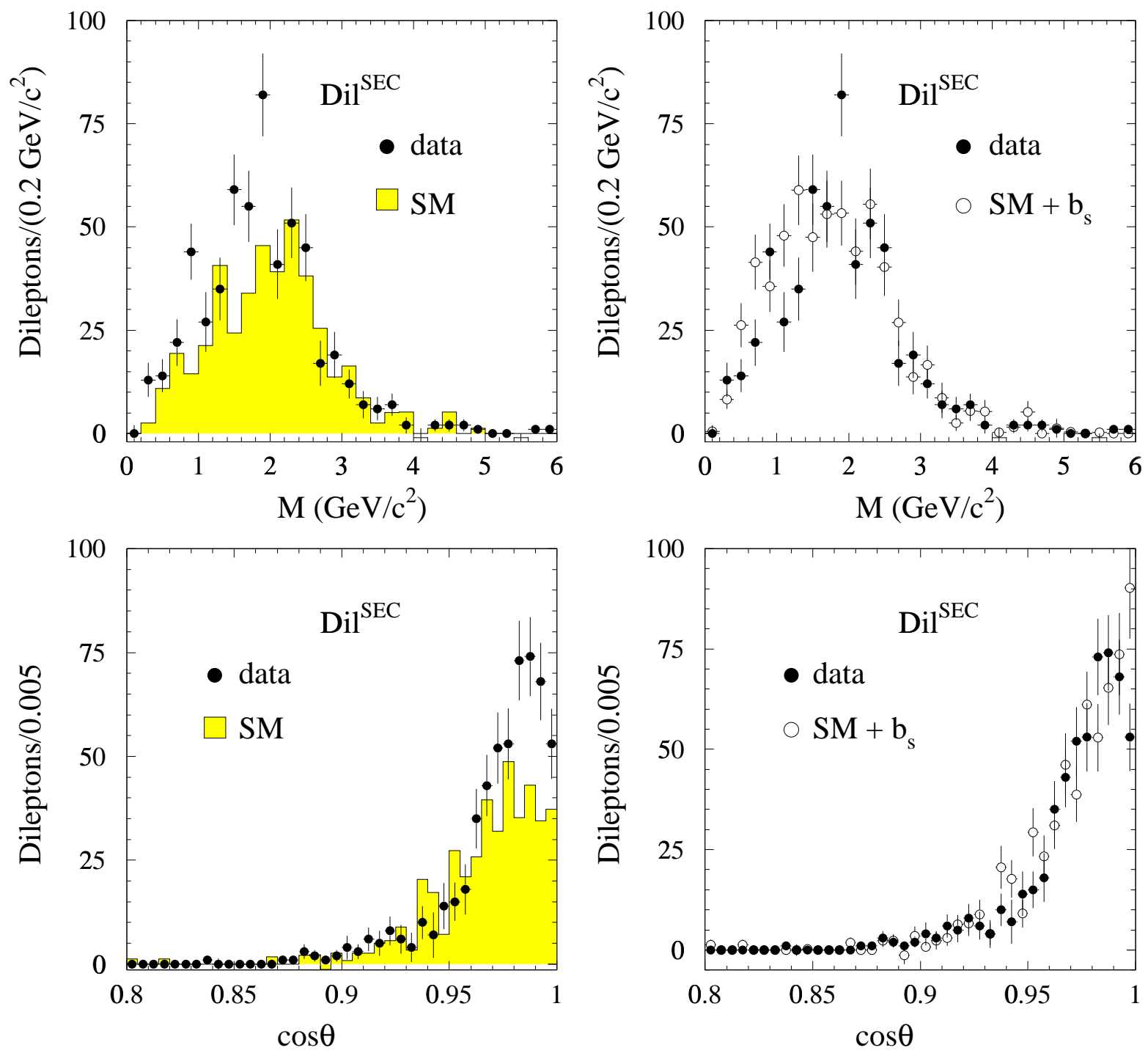

FIG. 3: Observed distributions of the invariant mass $M$ and the opening angle $\theta$ of lepton pairs contained in a jet tagged by the SECVTX algorithm are compared to the simulation.

present two additional tests of our model. 

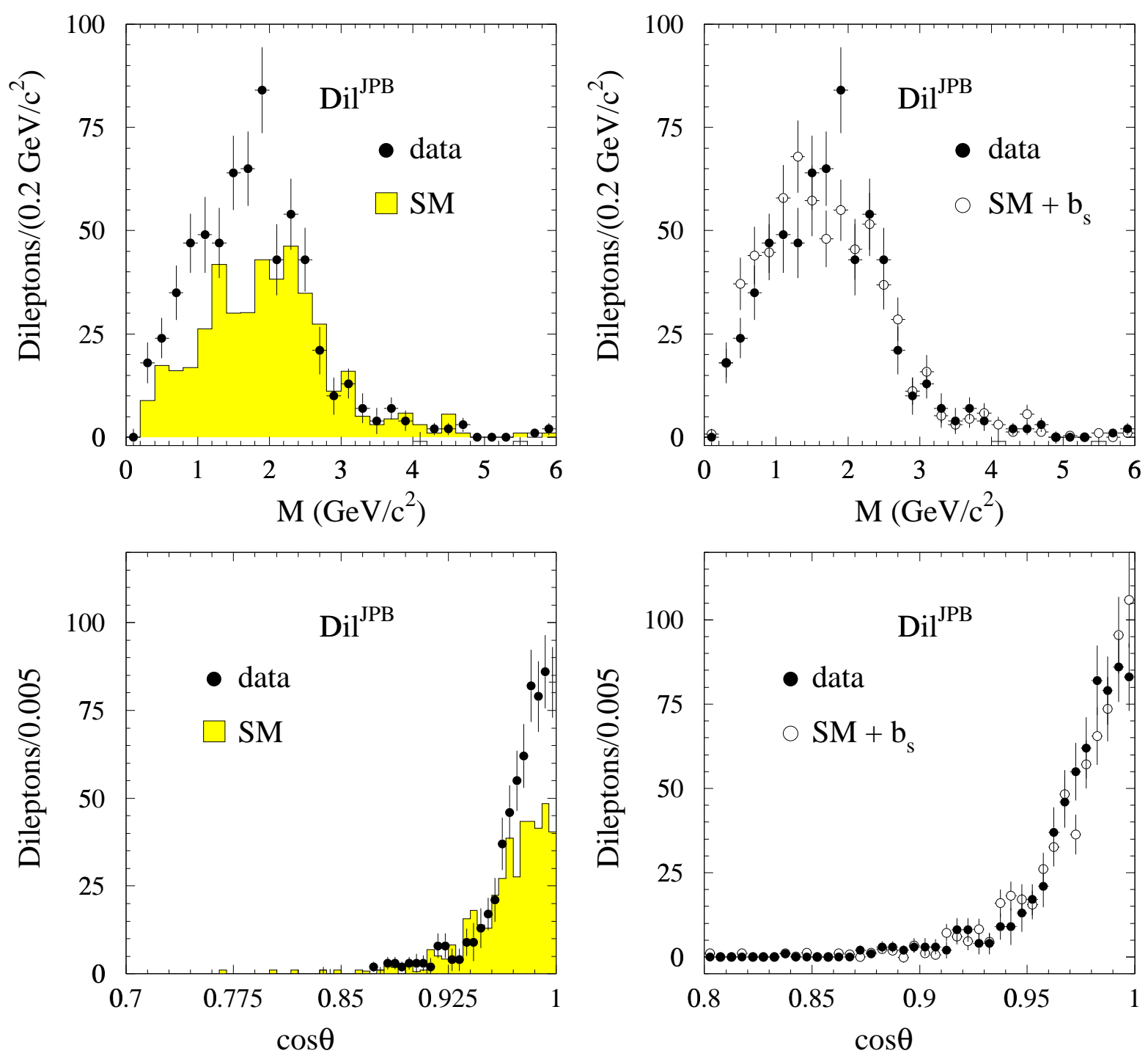

FIG. 4: Observed distributions of the invariant mass $M$ and the opening angle $\theta$ of lepton pairs contained in a jet tagged by the JPB algorithm are compared to the simulation.

\section{A. Study of the $l^{-} D^{0}$ system}

In Sec. X B of Ref. [23], the $b$ purity of the inclusive lepton sample has been verified by counting the number of $D^{0}$ mesons accompanying the trigger leptons. In the simulation, most of the $l^{-} D^{0}$ (and its charge conjugate) pairs arise from decays of single $b$ hadrons. 

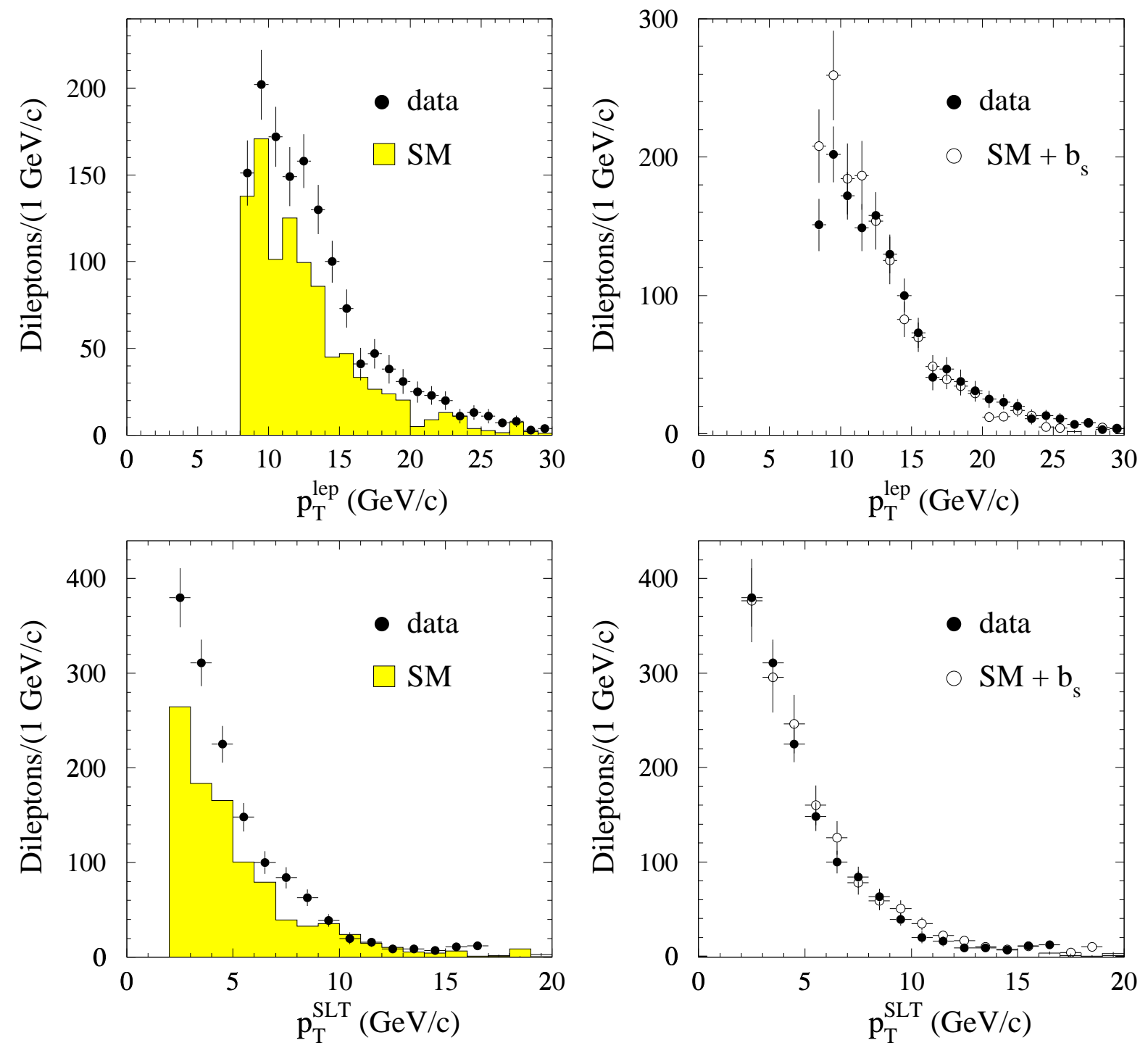

FIG. 5: Transverse momentum distributions of the trigger (lep) and soft (SLT) leptons contained in the same jet are compared to the simulation.

In that study, the ratio of the number of identified $l^{-} D^{0}$ pairs in the data to that in the $\mathrm{SM}$ simulation is $0.92 \pm 0.13$. This agreement has favored the initial assumption that $b_{s}$ quarks decay into $c$ quarks because our model replaces a fraction of the $b \bar{b}$ production with $b_{s} \bar{b}_{s}$ production. We search the $\mathrm{SM}+b_{s}$ simulation for $D^{0} \rightarrow K^{-} \pi^{+}$candidate around the direction of the trigger lepton following the same procedure described in Sec. X B of Ref. [23]. 
In the mass range of $1.82-1.92 \mathrm{GeV} / \mathrm{c}^{2}$ we identify $147 \pm 15 D^{0}$ mesons (after subtracting a background of $47 \pm 6$ events), in agreement with the number $126 \pm 15$ found in the data (after removing a background of $79 \pm 6$ events). In the $\mathrm{SM}+b_{s}$ simulation $24 \%$ of the $l^{-} D^{0}$ pairs arise from $b_{s}$ decays. Since the $b_{s}$ quark is assumed to be lighter than a $b$ quark, $l^{-} D^{0}$ pairs from $b_{s}$ decays should cluster at smaller invariant masses than those from $b$ decays. It is therefore of interest to compare the $l^{-} D^{0}$ invariant mass distribution in the data and the simulation (see Fig. 6). In this comparison the shape of the background is evaluated by using wrong-sign combinations $\left(l^{-} K^{+} \pi^{-}\right)$with the $K^{+} \pi^{-}$invariant mass in the range $1.82-1.92 \mathrm{GeV} / \mathrm{c}^{2}$. Figure $7(\mathrm{a})$ compares $l^{-} D^{0}$ invariant mass distributions in the data and in the simulation after background removal ${ }^{6}$.

A comparison of the two distributions in the mass range $2.4-5.2 \mathrm{GeV} / \mathrm{c}^{2}$ yields a $\chi^{2}$ of 10 for 13 DOF. However, the SM part of the simulation contains $112 \pm 14 l^{-} D^{0}$ candidates, which is also in agreement with the data (126 \pm 15 candidates). The invariant mass distribution of the $l^{-} D^{0}$ candidates contributed by the SM part of the simulation, shown in Figure 7(b), also agrees with the data (the $\chi^{2}$ of the comparison of the invariant mass distributions is 6 for $13 \mathrm{DOF})$. In the limited statistics of this sample, the $l^{-} D^{0}$ data do not provide additional support to our model.

\footnotetext{
${ }^{6}$ Because of the $p_{T}$ cuts used to select leptons, pions, and kaons, the $p_{T}$ threshold of the $l D^{0}$ system decreases rapidly with increasing $l D^{0}$ invariant mass. Therefore, the $l^{-} D^{0}$ invariant mass distribution is also affected by the $p_{T}$ distribution of the $\tilde{B}$ mesons that in turn is affected by the shortcomings of the HERWIG fragmentation and hadronization model (see Appendix B).
} 

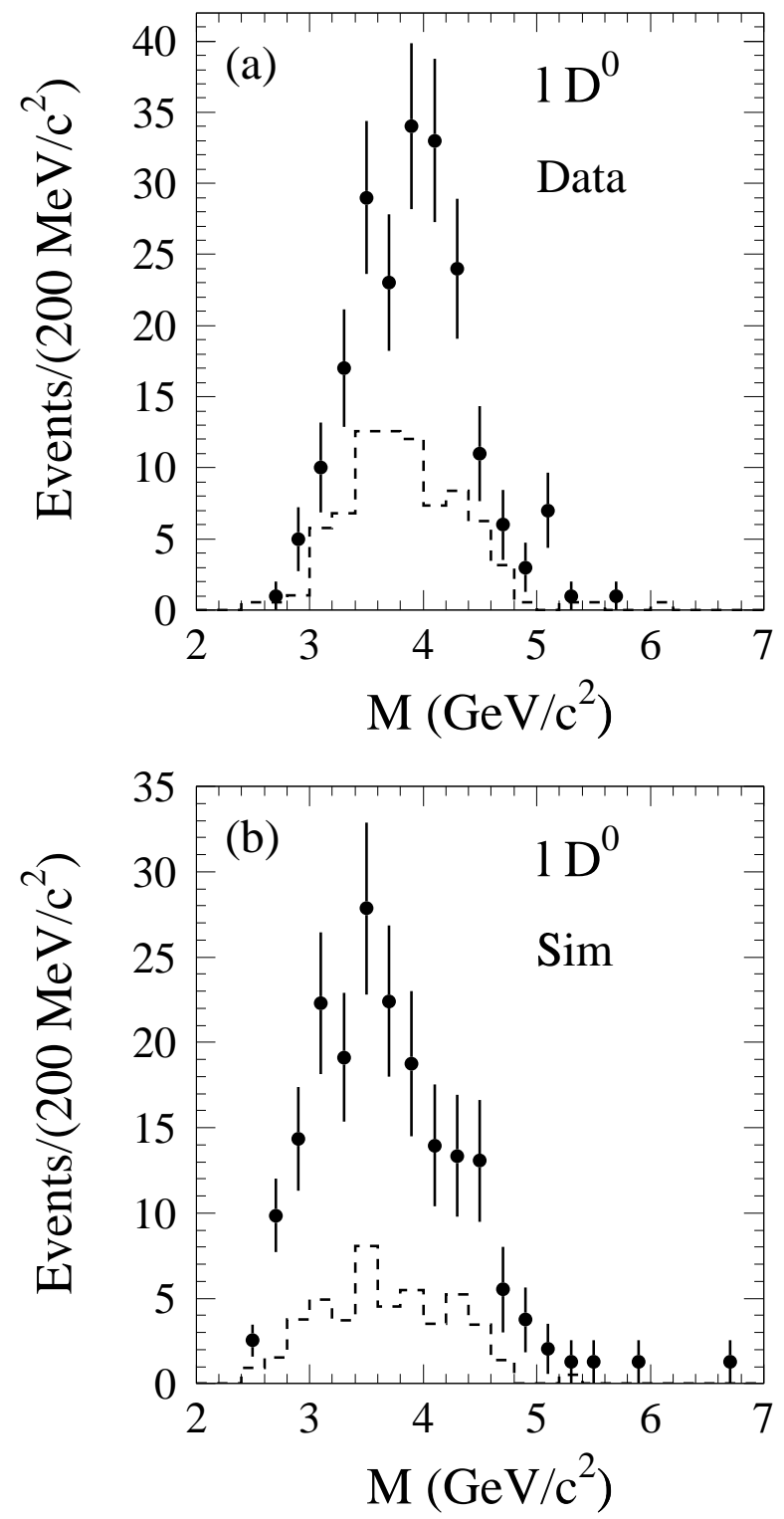

FIG. 6: Invariant mass distributions of $l^{-} D^{0}$ candidates in (a) the data and (b) the $\mathrm{SM}+b_{s}$ simulation. The dashed histograms represent the background evaluated using wrong-sign combinations (see text). 
TABLE III: Numbers of $J / \psi$ mesons in the data and in the $\mathrm{SM}+b_{s}$ simulation.

\begin{tabular}{lcccr}
\hline \hline & \multicolumn{2}{c}{$J / \psi \rightarrow e^{+} e^{-}$} & \multicolumn{2}{c}{$J / \psi \rightarrow \mu^{+} \mu^{-}$} \\
& data & simulation & data & simulation \\
$D i l_{\psi}$ & $176.0 \pm 14.4$ & $131.4 \pm 18.7$ & $83.0 \pm 9.4$ & $48.3 \pm 8.6$ \\
$D i l_{\psi}^{S E C}$ & $57.8 \pm 8.8$ & $61.3 \pm 9.5$ & $31.9 \pm 5.8$ & $25.7 \pm 5.2$ \\
$D i l_{\psi}^{J P B}$ & $61.2 \pm 8.4$ & $57.5 \pm 8.3$ & $29.6 \pm 5.7$ & $28.1 \pm 5.2$ \\
\hline \hline
\end{tabular}

\section{B. Rates of lepton-jets containing $J / \psi$ mesons}

$J / \psi$ mesons are produced in the decay of $B$ hadrons but not in the decay of $\tilde{B}$ hadrons with a $100 \%$ semileptonic branching ratio. The $\mathrm{SM}+b_{s}$ simulation predicts a rate of $J / \psi$ mesons from $B$ decays that is approximately $20 \%$ smaller than that predicted by the SM simulation (see Table XIV of Ref. [23]). Rates of $J / \psi$ in the data and in the $\mathrm{SM}+b_{s}$ simulation are listed in Table III. Rates of $J / \psi$ mesons contained in lepton-jets with SECVTX and JPB tags arise only from $B$ decays, and are well predicted by the $\mathrm{SM}+b_{s}$ simulation. Before tagging, $(50 \pm 5) \%$ of the $J / \psi$ mesons identified in the data (first row of Table III) do not arise from $B$ decays $[23,38]$. The $\mathrm{SM}+b_{s}$ prediction $(180 \pm 20)$ is still slightly larger than the data in which $130 \pm 11 \mathrm{~J} / \psi$ mesons originate from $B$ decays.

\section{LIFETIME}

In this section we use several techniques to estimate the lifetime of the object producing the excess of soft lepton tags with respect to the SM simulation. We extract the lifetime by a data to simulation comparison of the distributions of the following quantities strictly related to the lifetime:

1. $\tau_{\text {dil }}=\frac{L_{x y} M}{c p_{T}}$, where $M$ and $p_{T}$ are the invariant mass and transverse momentum of a lepton pair (trigger lepton plus soft lepton), and $L_{x y}$ is the projection on the $p_{T}$ direction of the distance between the primary vertex of the event and the vertex determined by the lepton tracks.

2. $\tau_{1}=\frac{4 \vec{d} \cdot \vec{p}_{T}}{\pi c\left|p_{T}\right|}$, where $\vec{d}$ is the vector corresponding to the distance of closest approach of 

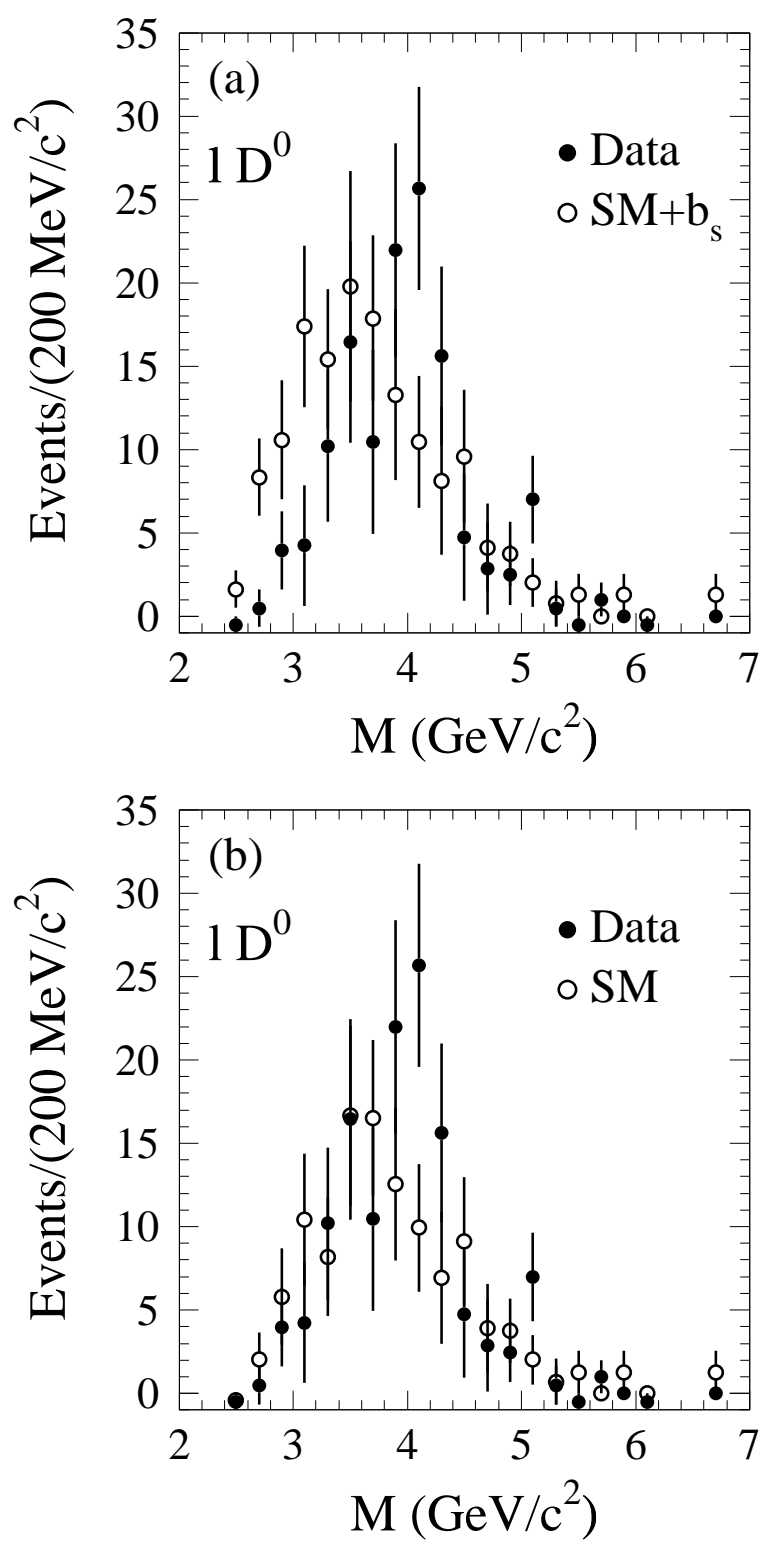

FIG. 7: The distribution of the $l^{-} D^{0}$ invariant mass after background removal is compared to the prediction of (a) the $\mathrm{SM}+b_{s}$ simulation and (b) the SM contribution only. 
a lepton track to the primary event vertex, and $\vec{p}_{T}$ is the transverse momentum of the jet containing a trigger (lep) or soft (SLT) lepton.

We test these methods using lepton pairs consistent with $J / \psi$ mesons produced by $b$ decays $\left(D i l_{\psi}^{J P B}\right.$ in Table III). Figure 8 shows agreement between data and simulation within the limited statistics of the $J / \psi$ sample.
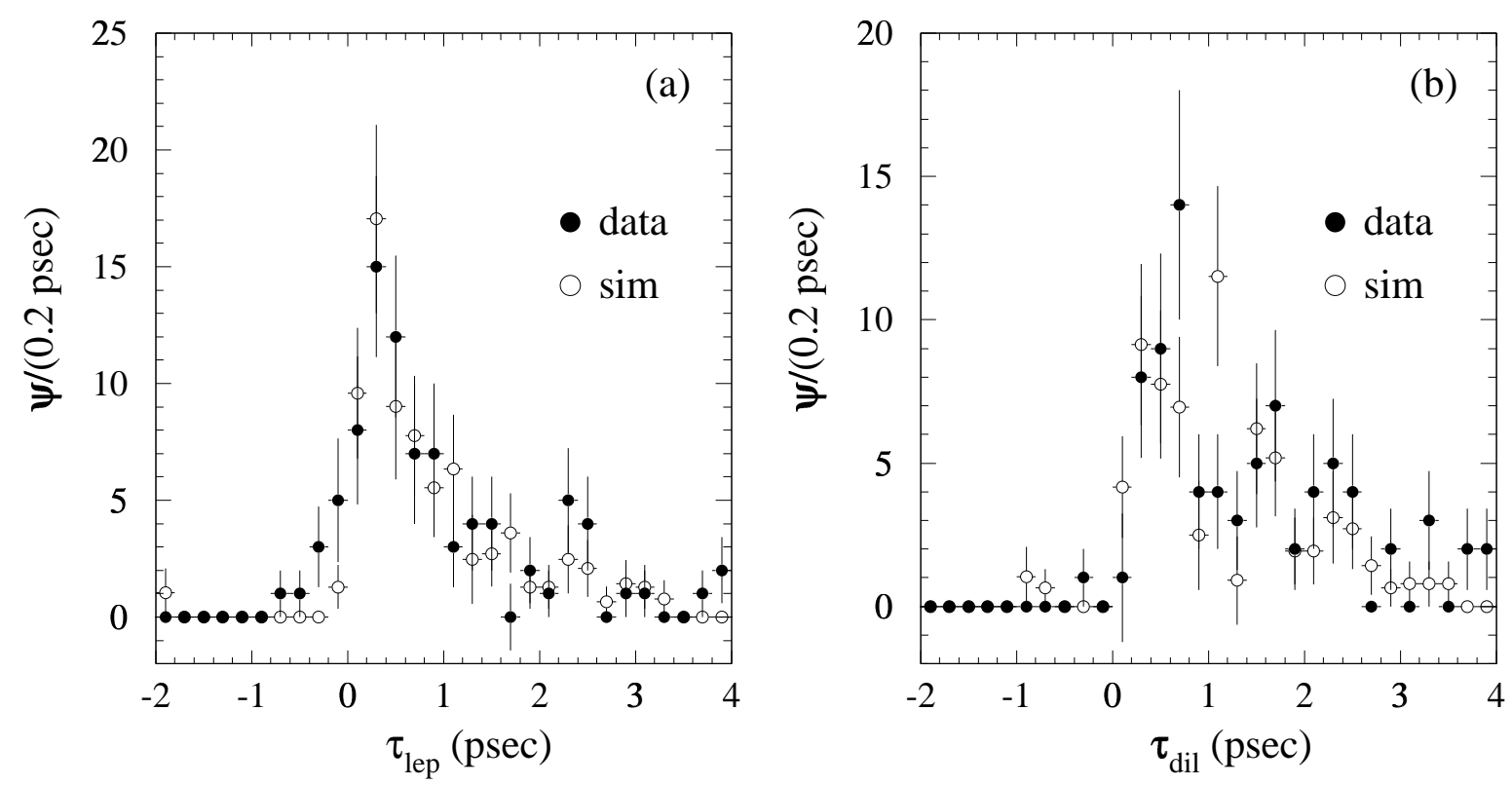

FIG. 8: Test of the distributions used to estimate the $b_{s}$ lifetime using $J / \psi$ mesons contained in a jet tagged by the JPB algorithm (see text).

Figures 9 and 10 compare lifetime distributions in the data and in the simulation implemented with the production of a scalar quark with a 1.0 ps lifetime and normalized as in Table I. Using the track parameters of the trigger and soft leptons in the same jet we construct the quantities $\tau_{\text {dil }}$ and $\tau_{\text {lep }}$. Using soft leptons in away-jets with JPB tags, we construct the quantity $\tau_{\mathrm{SLT}}$. In the data this last distribution has been obtained after subtracting the expected amount of fake SLT tags. The $\tau_{\text {SLT }}$ distribution for fake tags is approximated using the distribution of all SLT candidate tracks in these jets and is shown in Fig. 11. For each distribution we evaluate the quantity

$$
\chi^{2}=\sum_{i} \frac{(D(i)-S(i))^{2}}{E D(i)^{2}+E S(i)^{2}}
$$



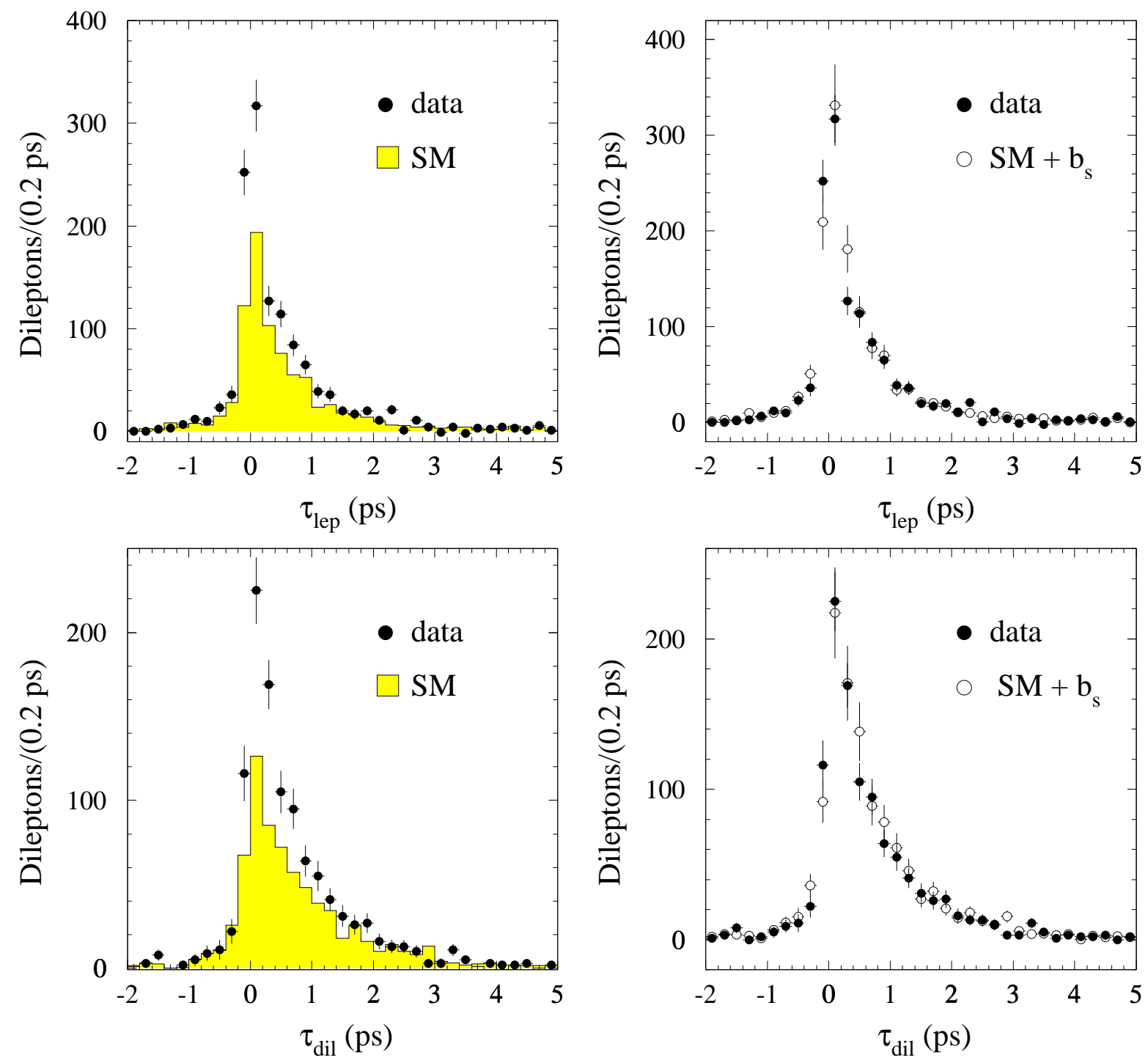

FIG. 9: Lifetime distributions derived using lepton pairs in the same jet are compared to the prediction of the simulations without (SM) and with $b_{s}$ production. The simulation is normalized according to the fit result listed in Table I. A comparison of the measured and predicted $\tau_{\text {lep }}$ distributions yields a $\chi^{2}$ of 8 for 15 DOF $\left(-1.0 \leq \tau_{\text {lep }} \leq 2 \mathrm{ps}\right)$; the comparison of the $\tau_{\text {dil }}$ distributions yields a $\chi^{2}$ of 8 for $12 \operatorname{DOF}\left(-0.4 \leq \tau_{\text {dil }} \leq 2 \mathrm{ps}\right)$. 

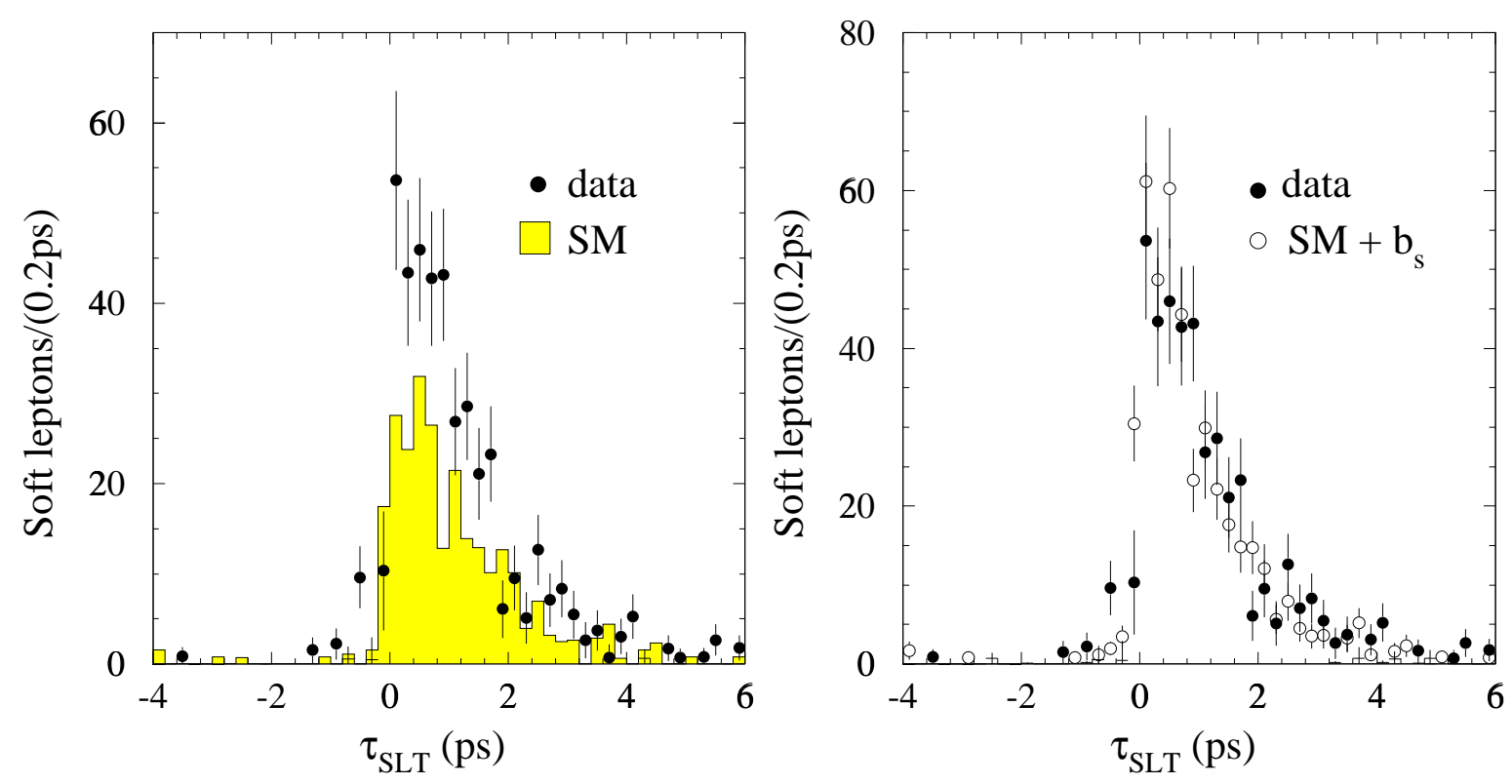

FIG. 10: Lifetime distributions derived using soft leptons contained in away-jets with JPB tags. The comparison of the $\tau_{\mathrm{SLT}}$ distributions in the data and the $\mathrm{SM}+b_{s}$ simulation yields a $\chi^{2}$ of 15 for $10 \mathrm{DOF}\left(0 \leq \tau_{\mathrm{SLT}} \leq 2 \mathrm{ps}\right)$.

where $D(i)$ and $S(i)$ are the contents of the $i^{\text {th }}$-bin of the measured and predicted distributions, respectively $\left(E D(i)\right.$ and $E S(i)$ are the corresponding errors). The $\chi^{2}$ is constructed avoiding large negative and positive tails which are not well modeled by our detector simulation [37]. We have produced a set of scalar quark simulations with lifetime values ranging from 0.2 to $3.0 \mathrm{ps}$ which we add to the SM simulation. We first fit each of these simulations to the data following the same procedure used in Sec. II. Since some tagging rates depend upon the lifetime, we use the $\chi^{2}$ value returned by the fit as an additional discriminant. The $\chi^{2}$ yields as a function of the $b_{s}$ lifetime are shown in Fig. 12 .

The fit of the measured tagging rate yields a lifetime of $0.54 \pm 0.3 \mathrm{ps}$

The $\tau_{\text {lep }}$ distribution yields a lifetime of $1.0 \pm 0.5 \mathrm{ps}$

The $\tau_{\text {dil }}$ distribution yields a lifetime of $0.9 \pm 0.6 \mathrm{ps}$

The $\tau_{\text {SLT }}$ distribution yields a lifetime of $1.55_{-0.4}^{+0.6} \mathrm{ps}$ 


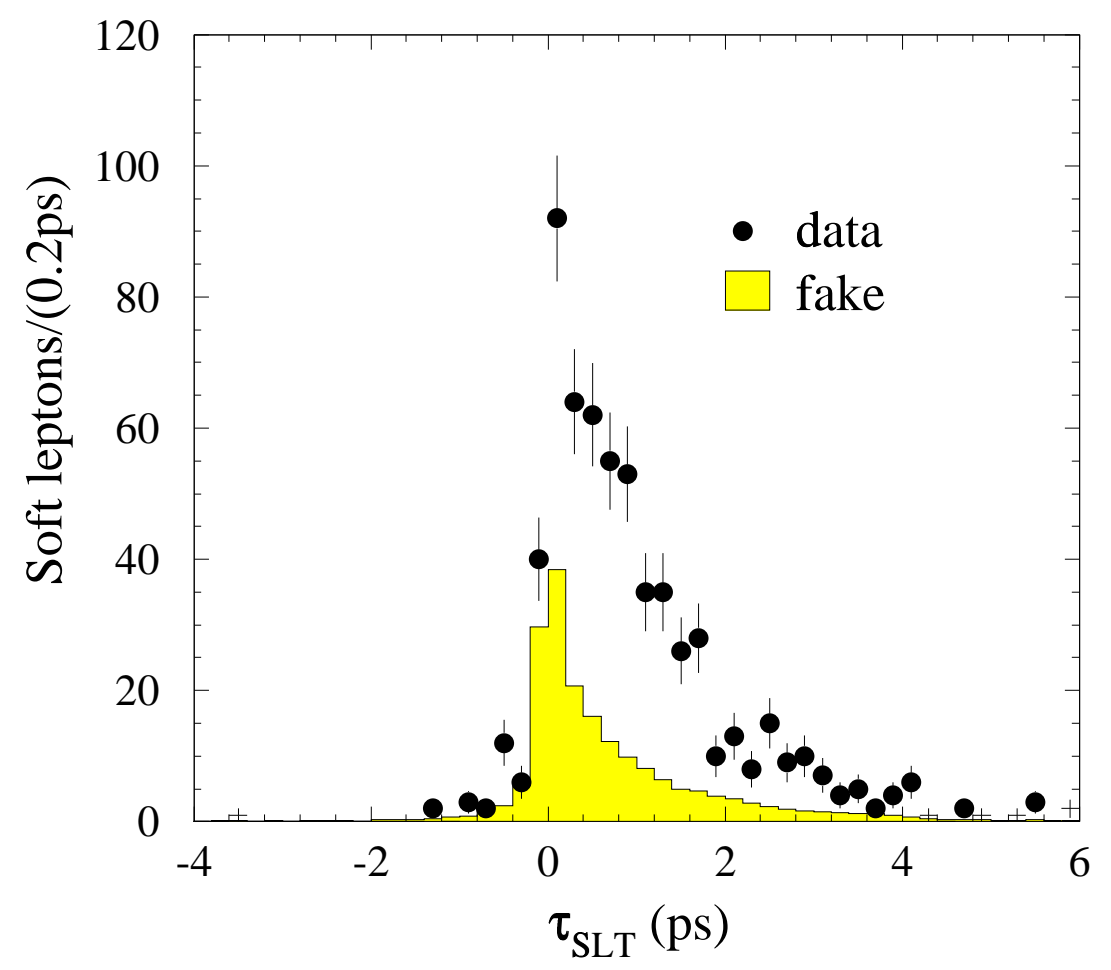

FIG. 11: The lifetime distribution of soft leptons in away-jets tagged by JPB is compared to the expected contribution of fake SLT tags.

The average of the four results is $1.0 \mathrm{ps}$; the uncertainty defined as the RMS deviation of the four measurements is $0.4 \mathrm{ps}$.

\section{CONCLUSIONS}

We review a number of known discrepancies [23-26, 29] between the heavy-flavor properties of jets produced at the Tevatron and the prediction of conventional-QCD simulations. These discrepancies may well be the result of larger than estimated experimental difficulties hidden in all $b \bar{b}$ cross section measurements [28]. However, in this study, we entertain the possibility that these effects are real, and investigate the constraints imposed by the data on the hypothesis that a fraction of the heavy flavor hadrons produced at the Tevatron might 

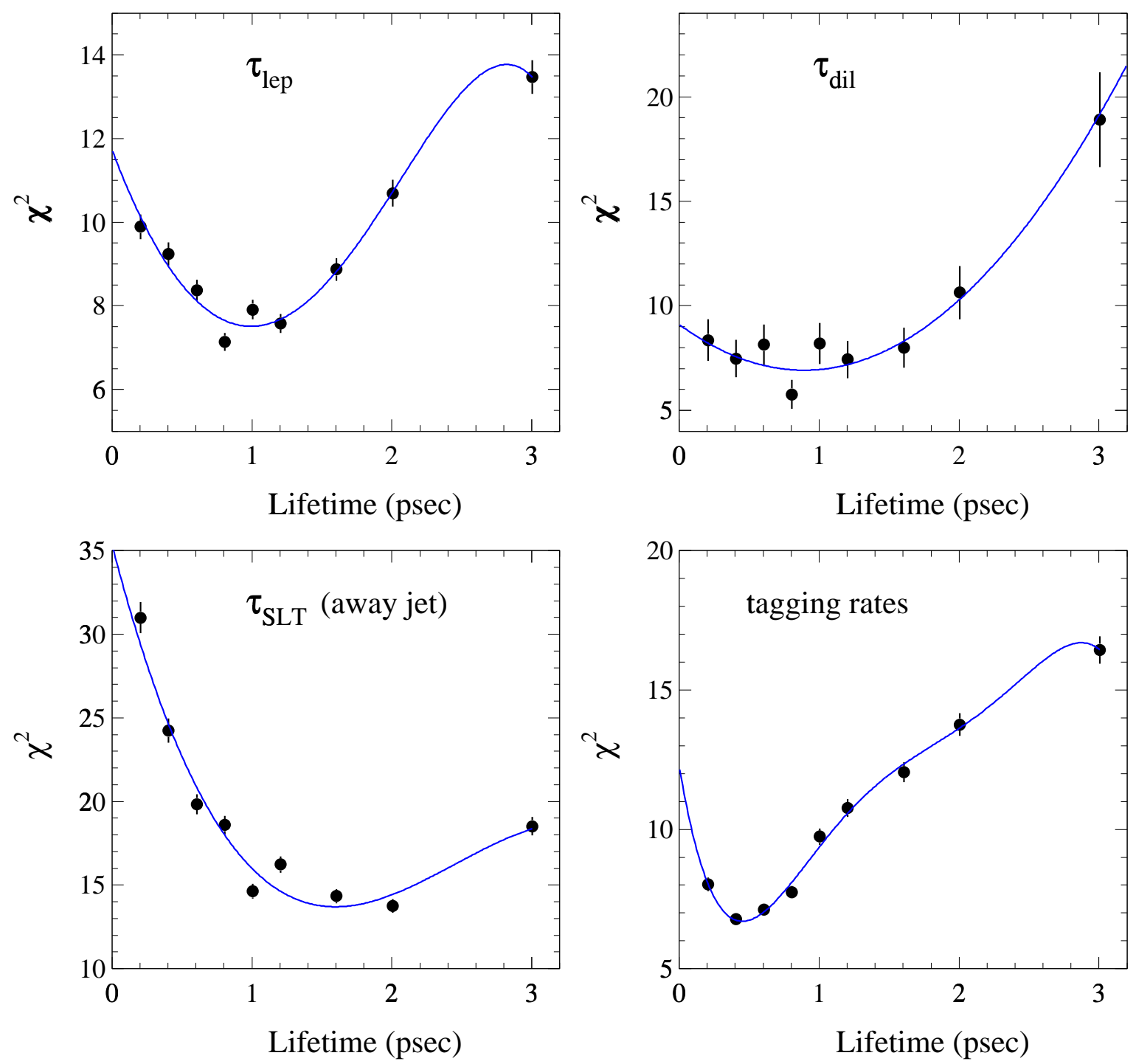

FIG. 12: Yields of $\chi^{2}$ resulting from comparisons of data and simulation as a function of the $b_{s}$ lifetime.

be due to unconventional sources. We postulate the existence of a sbottom quark $b_{s}$ that decays to a lepton, a charmed quark, and a neutral scalar non-interacting particle $\nu_{s}$ with a $100 \%$ semileptonic branching fraction, and implement its production into a conventionalQCD simulation based on the HERWIG Monte Carlo generator program. We initially use a $b_{s}$ mass of $3.6 \mathrm{GeV} / \mathrm{c}^{2}$ since it is not excluded in the study of Ref. [30], a massless $\nu_{s}$, a lifetime 
of $1 \mathrm{ps}$, and a decay mediated by the higgsino coupling to the right-handed matter. We use this generator to simulate the inclusive lepton sample collected by the CDF experiment in the 1992 - 1995 collider run. The data sample consists of events with two or more jets, one of which is required to contain a lepton with $p_{T} \geq 8 \mathrm{GeV} / \mathrm{c}$; this jet is referred to as lepton-jet, whereas jets recoiling against it are called away-jets. Following Ref. [23], this study uses rates of lepton- and away-jets containing displaced secondary vertices (SECVTX tags), tracks with large impact parameters (JPB tags), and soft ( $p_{T} \geq 2 \mathrm{GeV} / \mathrm{c}$ ) leptons (SLT tags) in order to determine the bottom, charmed, and $b_{s}$ content of the data. We

tune the parton-level cross section predicted by the simulation within the experimental and theoretical uncertainties. The best fit to the data returns a $b_{s}$ production cross section at the Tevatron that agrees with the NLO prediction for pair production of scalar quarks. According to the fit, the $b_{s}$ contribution to the inclusive lepton sample is approximately $23 \%$ (35\%) of that of the $b$ quark in the electron (muon) sample. This contribution models the invariant mass and opening angle distribution of lepton pairs contained in the same jet that were found anomalous when compared to a SM simulation in Ref. [29]. We have studied the sensitivity of the data to slightly different models and investigated the lifetime of the hypothetical $b_{s}$ quark. The best fit to the data replaces approximately $20 \%$ of the $b$ production with $b_{s}$ production. In our hypothetical model, the invariant mass distribution of $l D^{0}$ pairs coming from $b_{s}$ decays is expected to peak at smaller values than that of $l D^{0}$ pairs coming from $b$ decays. In the limited statistics of the data, we see no clear evidence of this. Since $b_{s}$ decays do not produce $J / \psi$ mesons, we have verified that rates of $J / \psi$ mesons in the data are consistent with those predicted by the simulation that includes $b_{s}$ production.

\section{ACKNOWLEDGMENTS}

We thank the Fermilab staff, the CDF collaboration, and the technical staff of its participating Institutions for their contributions. We thank M. Spira for making available the code of the PROSPINO Monte Carlo generator, and M. Mangano for working out many details of the matrix element used to decay sbottom quarks. This work was supported by the U.S. Department of Energy and the Istituto Nazionale di Fisica Nucleare. 


\section{APPENDIX A: DECAY $b_{\mathrm{s}} \rightarrow l c \nu_{\mathrm{s}}$}

The decay of $b_{s}$ hadrons is modeled using the spectator model (routine HWDHVY) available in the version 5.6 of HERWIG. In this model, HERWIG normally weights the phase space of the three-body semileptonic decay of a $b$ quark with the $\mathrm{V}$-A matrix element. In the case

of a scalar quark we have modified the HERWIG code and weight the phase-space with the matrix element:

$$
\frac{d \Gamma}{d z_{c} d z_{l}}=K\left[\left(1-z_{c}\right)\left(1-z_{l}\right)-R_{\nu_{s}}+R_{c}\left(z_{c}-z_{l}+R_{\nu_{s}}-R_{c}\right)\right]
$$

where $K$ is a normalization factor, $R_{c}=m_{c}^{2} / m_{b_{s}}^{2}$, and $R_{\nu_{s}}=m_{\nu_{s}}^{2} / m_{b_{s}}^{2}$.

Here $z_{c}$ and $z_{l}$ are defined as

$$
z_{c}=\frac{2 p_{b_{s}} \cdot p_{c}}{m_{b_{s}}^{2}} \quad \text { and } \quad z_{l}=\frac{2 p_{b_{s}} \cdot p_{l}}{m_{b_{s}}^{2}}
$$

The phase space limits are:

$$
\begin{gathered}
2 \sqrt{R_{c}}<z_{c}<1+R_{c}-R_{\nu_{s}} \\
\frac{1+R_{c}-R_{\nu_{s}}-z_{c}}{1-\left[z_{c}-\sqrt{z_{c}^{2}-4 R_{c}}\right] / 2}<z_{l}<\frac{1+R_{c}-R_{\nu_{s}}-z_{c}}{1-\left[z_{c}+\sqrt{z_{c}^{2}-4 R_{c}}\right] / 2}
\end{gathered}
$$

This matrix element for the decay $b_{s} \rightarrow c l \nu_{s}$ is derived from the tree level calculation outlined in Ref. [39]:

$$
\mathcal{M}=\sum_{j=1}^{2} \frac{-g^{2} V_{j 1} F_{L}\left|V_{c \tilde{b}}\right|}{\left(p_{\tilde{b}}-p_{c}\right)^{2}-m_{\chi_{j}}^{2}}\left[\left[U_{j 1} \cos \theta-\frac{m_{b} U_{j 2} \sin \theta}{\sqrt{2} m_{W} \cos \beta}\right] m_{\chi_{j}} \bar{u}\left(p_{c}\right) P_{R} v\left(p_{l}\right)+\frac{m_{c} V_{j 2}^{*} \cos \theta}{\sqrt{2} m_{W} \sin \beta} \bar{u}\left(p_{c}\right) \not_{\tilde{\nu}} P_{R} v\left(p_{l}\right)\right]
$$

where $\chi_{j}$ 's are the chargino mass eigenstates, and $U$ and $V^{*}$ are the mixing matrices for the right and left-handed charginos, respectively. The first subscript of $U, V$ corresponds to mass eigenstates and the second to weak eigenstates ( 1 for the gaugino and 2 for the higgsino). Here $\tan \beta$ is the ratio of the vacuum expectation values of the two Higgs fields, $\theta$ is the mixing angle between left-handed and right-handed scalar quarks, $F_{\mathrm{L}}$ is the fraction of left-handed component of the scalar neutrino, and $\left|V_{c \tilde{b}}\right|$ is the CKM matrix element. Equation (A1) follows from (A2) when the decay is mediated by the higgsino coupling to the right-handed matter. If the decay is mediated by the gaugino coupling to the left-handed matter the matrix element is

$$
\frac{d \Gamma}{d z_{c} d z_{l}}=K\left(z_{c}+z_{l}-1+R_{\nu_{s}}-R_{c}\right)
$$


In the latter case the two fermions in the final state are both left-handed and tend to be produced back-to-back since the initial state is spinless. Using equation (A1) the two fermions in the final state have opposite handedness and tend to be produced more collinear than when using equation (A3). It follows that the matrix element (A1) produces leptons with a momentum distribution appreciably softer than that produced by the matrix element (A3) or by a phase space decay; this latter mode has been used in Ref. [22] to search evidence for such a scalar quark.

The $c$ quark emerging from the $b_{s}$ decay is recombined with the spectator quark by the HERWIG spectator model. The decay of the resulting $c$ hadron is performed with the QQ Monte Carlo program. In the spectator model, excited $D$-meson states are produced only by the hadronic current carried by the virtual $W$ (so called upper vertex). Since we impose that the gauge fermion involved in the scalar quark decay has only leptonic decay modes, the simulation produces a very simple list of $c$ hadrons with respect to the QQ generator (see Fig. 13). The value of the $\tilde{B}$ masses returned by the spectator model, which does not have a look-up table similar to that for $B$ hadrons, are no more than an educated guess, probably with an uncertainty of a few hundred $\mathrm{MeV}$. The fractions of different $c$-hadrons produced by the spectator model are also quite arbitrary. 

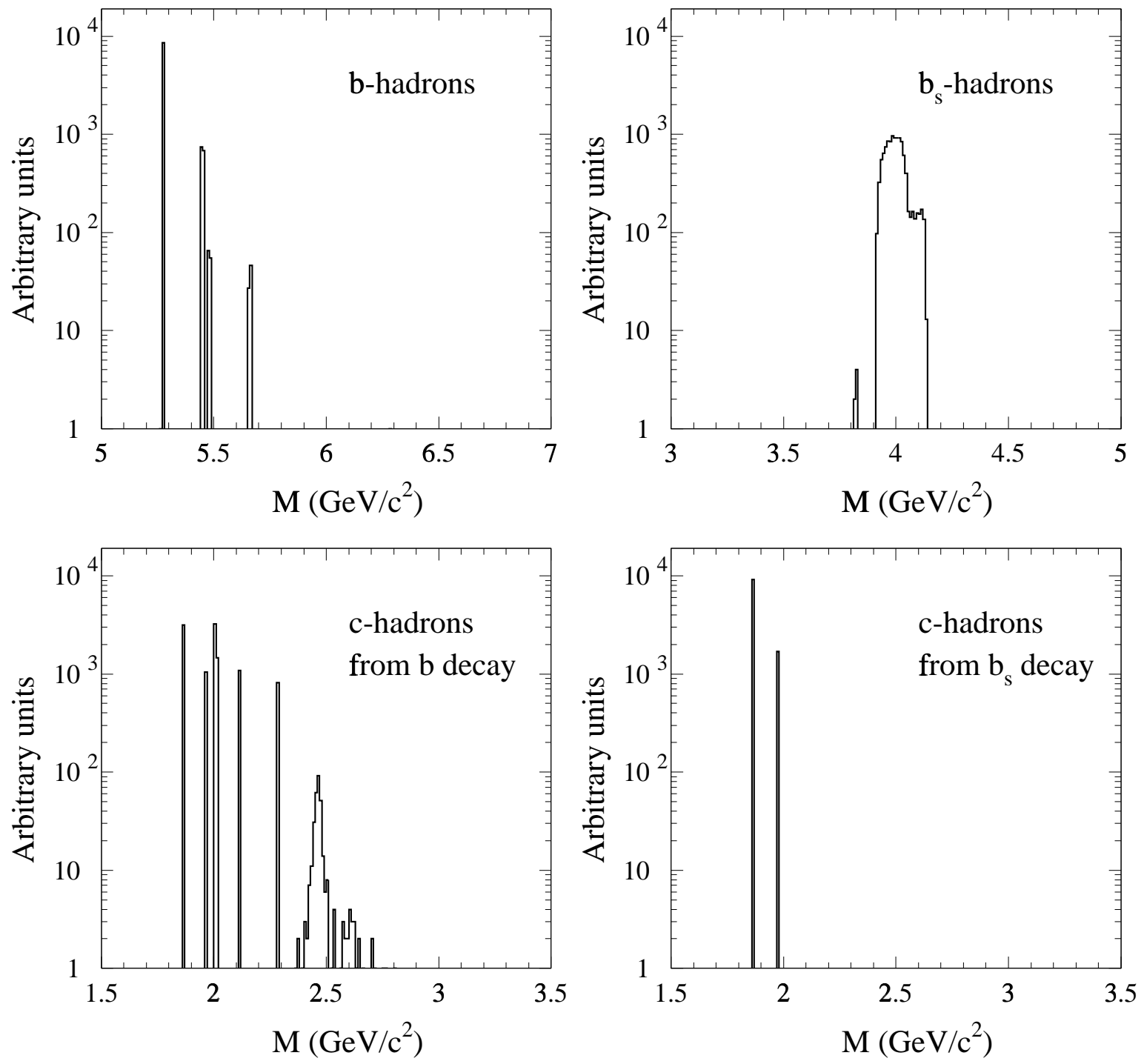

FIG. 13: Mass spectrum of: $b$ and $b_{s}$ hadrons formed by the HERWIG generator using $m_{b}=$ $4.75 \mathrm{GeV} / \mathrm{c}^{2}$ and $m_{b_{s}}=3.65 \mathrm{GeV} / \mathrm{c}^{2} ; c$ hadrons from $b$-hadron decays modeled with the QQ generator; and $c$ hadrons from $b_{s}$-hadron decays modeled with the HERWIG spectator model.

\section{APPENDIX B: MODELING OF THE $b_{s}$ PRODUCTION}

We implement the production of pairs of scalar quarks in $p \bar{p}$ collisions using option 1705

of the version 5.6 of the HERWIG generator program. This option generates $b \bar{b}$ events with 
LO and flavor excitation diagrams, but without gluon splitting diagrams. We use a $b$-mass value of $3.65 \mathrm{GeV} / \mathrm{c}^{2}$ and initially set the $b_{s}$ lifetime equal to $1.0 \mathrm{ps}$. The $B$ hadrons, formed at the end of the $b$-fragmentation process, are changed into fictitious $\tilde{B}$ hadrons and decayed by the HERWIG spectator model fed with the $b_{s} \rightarrow l c \nu_{s}$ matrix element described in the previous Appendix. The decay of the $c$-hadron formed by HERWIG using the spectator quark is modeled with the QQ generator program [33]. Our modeling of the $b_{s}$ production suffers of the following approximations:

1. The $b_{s} \bar{b}_{s}$ production cross section has not the same dependence on the velocity $\beta$ of the partons in the final state as the $b \bar{b}$ cross section [40]. However, we study jets with transverse energies larger than $15 \mathrm{GeV}$ and this alleviates the problem, since the corresponding partons have energies larger than $20 \mathrm{GeV}$.

2. The spectrum of gluon emission in the hadronization process of a spin 0 quark differs from that of a spin $1 / 2$ quark. However, the average energy loss due to perturbative gluon emission off a spin-0 and a spin-1/2 particle is believed to be small [41].

3. Option 1705 does not evaluate the gluon splitting contribution to $b_{s}$ production. However, we tune the $b_{s}$ production to reproduce the observed rates of tagged lepton- and away-jets. When only looking at yields of tagged lepton- and away-jets, Table X of Ref. [23] shows that events arising from flavor excitation and gluon splitting are quite similar. Therefore we fit the data with the SM simulation implemented with the scalar quark production leaving its direct production and flavor excitation cross sections as additional free fit parameters; in first approximation, the flavor excitation cross section determined by the fit also accounts for the gluon splitting contribution.

[1] D. Acosta et al., Phys. Rev. D 65, 052007 (2002).

[2] V. Abazov et al., Phys. Rev. Lett. 94, 152002 (2005).

[3] G. Apollinari et al., hep-ex/0109020.

[4] H. E. Haber and G. L. Kane, Phys. Rep. 117C, 76 (1985).

[5] C. Nappi, Phys. Rev. D 25, 84 (1982).

[6] M. Carena et al., Phys. Rev. Lett. 86, 4463 (2001). 
[7] S. Pacetti and Y. Srivastava, hep-ph/0007318.

[8] E. L. Berger et al., Phys. Rev. Lett. 86, 4231 (2001).

[9] U. Nierste and T. Plehn, Phys. Lett. B 493, 104 (2000).

[10] A. Dedes and H. E. Haber, JHEP0105, 006 (2001).

[11] T. Becher et al., hep-ph/0112129.

[12] G. Cho, Phys. Rev. Lett. 89, 091801 (2002).

[13] J. Cao, Z. Xiong and Y. M. Yang, Phys. Rev. Lett. 88, 111802 (2002).

[14] S. Baek, Phys. Lett. B 541,161 (2002).

[15] K. Cheung and W. Keung, Phys. Rev. Lett. 89, 221801 (2002).

[16] K. Cheung and W. Keung, Phys. Rev. D 67, 015005 (2003).

[17] C. Chang, J. Y. Cui and J. M. Yang, Commun. Theor. Phys. 39, 197 (2003).

[18] R. Malhotra and D. Dicus, Phys. Rev. D 67, 097703 (2003).

[19] E. L. Berger, G. T. Bodwin and J. Lee, Phys. Lett. B 552, 223 (2003).

[20] Z. Luo and J. L. Rosner, Phys. Lett. B 569, 194 (2003).

[21] P. Janot, Phys. Lett. B 594, 23 (2004).

[22] V. Savinov et al., Phys. Rev. D 63, 051101 (2001).

[23] D. Acosta et al., Phys. Rev. D 69, 072004 (2004).

[24] F. Abe et al., Phys. Rev. D 53, 1051 (1996).

[25] F. Abe et al., Phys. Rev. D 55, 2546 (1997).

[26] B. Abbot et al., Phys. Lett. B 487, 264 (2000).

[27] T. Shears, "Charm and Beauty Production at the Tevatron", talk presented at the Int. Europhys. Conf. on High Energy Phys., Lisboa, Portugal (2005);

http : //www.lip.pt/events/2005/hep2005/talks/hep2005_talk_TaraShears.ppt.

[28] F. Happacher et al., hep-ph/0509348.

[29] G. Apollinari et al., Phys. Rev. D 72, 072002 (2005).

[30] G. Apollinari et al., Phys. Rev. D 72, 092003 (2005).

[31] D. G. Aschman et al., Phys. Rev. Lett. 39, 124 (1977); A. M. Boyarski et al., Phys. Rev. Lett. 34, 762 (1975). R. F. Schwitters in Proceedings of the XVIII International Conference on High Energy Physics, Tbilisi (1976), edited by N. N. Bogoliubov et al., (JINR, Dubna, U.S.S.R. (1977).

[32] G. Marchesini and B. R. Webber, Nucl. Phys. B 310, 461 (1988); G. Marchesini et al., 
Comput. Phys. Commun. 67, 465 (1992).

[33] Version 9_1 of the CLEO simulation; P. Avery, K. Read, G. Trahern, Cornell Internal Note CSN-212, March 25, 1985 (unpublished).

[34] D. Buskulic et al., Phys. Lett. B 313, 535 (1993).

[35] W. Beenakker et al., Nucl. Phys. B 492, 51 (1997); hep-ph/9611232.

[36] M. L. Mangano, P. Nason and G. Ridolfi, Nucl. Phys. B 373, 295 (1992). The Fortran code was made available by the authors.

[37] T. Affolder et al., Phys. Rev. D 64, 032002 (2001); Erratum-ibid. D 67, 119901 (2003).

[38] F. Abe et al., Phys. Rev. Lett. 79, 572 (1997); Phys. Rev. Lett. 79, 578 (1997).

[39] H. Hikasa and M. Kobayashi, Phys. Rev. D 36,724 (1987); J. F. Gunion and H. E. Haber, Nucl. Phys. B 272, 1 (1986).

[40] W. Beenakker et al., Nucl. Phys. B 515, 3 (1998).

[41] W. Beenakker, R. Hopker and P. M. Zerwas, Phys. Lett. B 349, 463 (1995). 